\title{
Review
}

\section{Clinical Metritis and Endometritis in Diary Cattle: A Review}

\author{
Kassahun A. Negasee, DVM, MSc* \\ Department of Animal Health, Awi Nationalities Administrative Zone Livestock Resource Development Office,Amhara Region, Ethiopia

\section{*Corresponding author} \\ Kassahun A. Negasee, DVM, MSc \\ Doctor, Department of Animal Health, Awi Nationalities Administrative Zone Livestock Resource Development Office,Amhara Region, Ethiopia; \\ E-mail: kassahunayana@gmail.com
}

\section{Article information}

Received: June 2 $2^{\text {nd }}, 2020$; Revised: August 18 ${ }^{\text {th }}, 2020$; Accepted: September 30 $0^{\text {th }}, 2020$; Published: November $5^{\text {th }}, 2020$

\section{Cite this article}

Negasee KA. Clinical metritis and endometritis in diary cattle:A review. Vet Med Open J. 2020; 5(2): 5 I-56. doi: 10. I7I40/VMOJ-5-I49

\begin{abstract}
Reproduction is one of the key pillars of production in many herds and individual animals. In female animals, reproduction can be affected by uterine diseases including metritis and endometritis. The objective of this review is to understand metritis and endometritis, diagnosis and treatment approaches, and the economic impact of uterine inflammations. Metritis is inflammation of the wall of the uterus, whereas endometritis is inflammation of the functional lining of the uterus, called the endometrium. Clinically, they are characterized by an enlarged uterus containing a watery red-brown fluid to viscous off-white purulent uterine discharge, which often has a fetid odor within 10 to 14-days after postpartum period in clinical metritis whereas clinical endometritis can also be observed its clinical signs after 21-days postpartum period. The risk factors for the occurrence of clinical metritis and endometritis include extrinsic factors include calving season and nutrition whereas intrinsic factors include retained fetal membrane (RFM), dystocia, metabolic disturbance, negative energy balance (NEB) and other non-specific diseases. These factors disturbing the normal physiology and weaken the immune system of dairy cattle. The factors favor the multiplication of pathogenic bacteria and cause inflammation of the uterus and results in metritis and endometritis. This result in increased conception interval increase the number of inseminations, loss of milk and meat production, marked drop in fertility and increase the cost of treatment. Clinical metritis and endometritis can be diagnosed by clinical symptoms and cytological examinations. Clinical metritis and endometritis can be treated by anti-inflammatory drugs flunixin meglumine, antibiotics (pens-trip and long-acting oxytetracycline), intrauterine infusion by using saline or iodine tincture solutions. Clinical metritis and endometritis are the most frequent uterine disorders in dairy cows causing decreased fertility leading to high economic losses. Therefore, management of these production diseases can be achieved by early diagnosis and treatment, supplementation of balanced feed containing minerals, vitamins, appropriate proteins and carbohydrates, good quality management during and after calving of the female animals.
\end{abstract}

\section{Keywords}

Diagnosis; Dairy; Disease; Clinical; Endometritis; Metritis; Risk factors; Therapeutics.

\section{Abbreviations}

LPS: Lipopolysaccharide; NEB: Negative energy balance; NSAIDs: Non-steroidal anti-inflammatory drugs; PGF2 $\alpha$ : Prostaglandin F2 alpha; PMNs: Polymorpho neutrophil; RFM: Retained fetal membrane.

\section{INTRODUCTION}

$\mathrm{R}$ eproduction is one of the key pillars of production in many herds and individual animals. ${ }^{1}$ It could be affected by reproductive diseases that decrease fertility in female animals includes metritis, endometritis, pyometra, retained fetal membrane (RFM) and some non-specific infections of the uterus are the most important factors causing infertility in the dairy cattle. ${ }^{2}$ The most important causes of infertility in dairy cows are reported to be metritis and endometritis. ${ }^{3}$

Many postpartum reproductive disorders have common etiology and predispose to each other and share a common therapeutics regime. ${ }^{3}$ Among the postpartum, reproductive diseases include metritis and endometritis. Metritis is inflammation of the wall of the uterus, whereas endometritis is inflammation of the 
functional lining of the uterus, called the endometrium. ${ }^{4}$

Metritis and endometritis have wide variations. ${ }^{5}$ Inflammation of uterus occurred after entry of bacteria during or immediately after parturition, coitus or while carrying out artificial insemination. Severity and persistence of infection of uterus depends on the degree of contamination, weak uterine defense mechanism and presence of substrates for the growth of the microbes such as devitalized tissues are the factors for the occurrence of uterine diseases. ${ }^{6}$ Pathogenic microbial infections of uterus that cause uterine disease are common in modern dairy cattle after parturition and lead to decreased productivity and sub-fertility from metritis and endometritis.?

Clinically metritis and endometritis can be diagnosed based on clinical symptoms, manage mental history during parturition, detecting vaginal discharge with the aid of speculum or gloved hands and cytological examinations give information about their diagnosis. The uterine diseases can be managed by intrauterine wash, systemic antibiotics, and hormones. ${ }^{8}$ In more severe cases other symptomatic therapies like anti-inflammatory agents and intravenous fluid therapy is also advocated.'

Inflammation of the uterus slows down the process of involution in the uterus and delays the onset of activities of the ovaries leading to economic loss due to systemic illness, increases conception interval, increases the number of inseminations, loss of milk and meat production and marked drop fertility.,3 Metritis and endometritis are the most important and dramatic diseases that disrupt the reproductive performance of cows and reduced livestock profitability. ${ }^{5}$ Therefore, this is to know the etiology, risk factors, pathophysiology, diagnostic, treatment, and economic impact of clinical metritis and endometritis in dairy cattle.

\section{CLINICAL METRITIS AND ENDOMETRITIS}

\section{Clinical Metritis}

Metritis is inflammation of the wall of the uterus. It is a postpartum infection of the uterus ${ }^{6}$ and it is the inflammation of the uterus consisting of both the endometrial and the muscular layer. ${ }^{9}$ Most of the cases occurred during the first 10-14-days of delivery and sometimes it is referred as toxic puerperal metritis ${ }^{2,6}$ or pelvic inflammatory disease (PID). ${ }^{4}$ It is characterized by an enlarged uterus containing a watery red-brown fluid to viscous off-white purulent uterine discharge, which often has a fetid odor or unpleasant smell. ${ }^{10}$

\section{Endometritis}

Endometritis is inflammation of the functional lining of the uterus, called the endometrium. ${ }^{4,8}$ It also refers to inflammation of the mucus membrane of the uterus; it is characterized by purulent discharge from the vulva that appeared three weeks after parturition or later. ${ }^{11}$ Clinical endometritis is defined as the presence of a purulent discharge detectable in the vagina 21-days or more postpartum or mucopurulent discharge detectable in the vagina after 26-days of postpartum. ${ }^{10}$ Subclinical endometritis is characterized by inflammation of the endometrial and the presence of neutrophils in cytology or biopsy histology, in the absence of signs of clinical endometritis. ${ }^{10,11}$

\section{Risk Factors and Occurrence of Clinical Metritis and Endometritis}

Clinical metritis and endometritis are considered to be a multifactorial disease with many factors having a direct or indirect effect on uterine function. ${ }^{4}$ Risk factors were categorized as extrinsic and intrinsic factors. Extrinsic factors concerned all conditions and environmental characteristics shared by animals in same herd, whereas intrinsic factors are specific variable characteristics of the individual cow. ${ }^{4,5}$

\section{Extrinsic factors}

Calving season: During the rainy seasons, the general health of cows decreases making them more vulnerable to uterine infections because the environment is contaminated by animal products and wastes. The wet season is good for multiplication of the pathogenic bacteria and the pathogens during parturition can enter to reproductive organ and causes uterine diseases like metritis and endometritis. $^{5}$

Nutrition: The quantity and quality of proteins included in the food ration play a key role in the efficiency of the immune system. Cellular immunity is also affected by protein quantity in food rations; protein deficiency dramatically reduces cellular phagocytosis. Noteworthy also is that excess protein intake induces high serum ammonia, which reduces lymphocyte production and favors the development metritis and endometritis. ${ }^{5,12}$

Feeding animals that contain many vitamins are involved in the functioning of the immune system, including vitamins $B$ and $\mathrm{C}$, which have a role in antibody synthesis, and vitamin $\mathrm{C}$, which ensures integrity of immune cell membranes and protects them from free radicals. Vitamin A is important for epithelial tissue development and cell differentiation, which are very important in the early postpartum stage. Vitamin A also has immunological functions by increasing the early inflammatory phase through enhancing the number of macrophages at the site of a wound, which improves localization and stimulation of the immune response in the case of uterine infection. Therefore, deficiency of vitamins during the postpartum period delays phagocytosis and favors for multiplication of pathogenic micro-organisms in the uterus reproductive organs, and other tissues that cause uterine diseases including clinical metritis and endometritis. ${ }^{5}$

A variety of minerals in the feed of female animals are involved in the uterine defense mechanisms. Calcium acts as complement-activator and calcium deficiency delays uterine involution and prolongs uterine infection. Calcium deficiency may be induced by excessive intake of phosphorus. Magnesium is involved in opsonization mechanisms. Selenium is involved in neutrophil function and its deficiency disrupts the reproductive performance of the cow and increases the risk of endometritis. Copper, zinc, and iron intervene in lysosome production and their deficiency dra- 
matically reduces phagocytosis and favors the growth of bacteria and development of metritis and endometritis., ${ }^{5,12}$

\section{Intrinsic factors}

Parity of the animals: As parity increases cows are often subject to dystocia, uterine lesions could be occurred and this causes uterine diseases including metritis and endometritis related to the severity of initial uterine lesions. Older cows have reduced uterine elasticity and uterine involution is slower than in younger cows, which presumably increases the vulnerability of older cows to persistent uterine infection and endometritis. ${ }^{5}$

In cases of bacterial contamination, older cows may efficiently and rapidly eliminate bacteria from the uterus while younger cows may have limited prior exposure, which delays the immune response, resulting in more severe and prolonged clinical and subclinical endometritis. ${ }^{5,10}$

Dystocia: Dystocia is a risk factor that significantly increased the overall incidence of clinical metritis and endometritis after calving. Dystocia indirectly increases the opportunity for the development of endometritis by increasing the probability of metritis. ${ }^{5} \mathrm{Ab}$ normal calving and dystocia can induce trauma on endometrium and calving assistance favors the introduction of bacteria into the uterus and increases the potential for clinical and subclinical endometritis to develop. ${ }^{5,13}$

Retained placenta: Retained placental tissue represents the main important risk factor for metritis and endometritis in dairy cows. Residual tissue encountered in the case of a retained placenta represents a favorable medium for the growth of bacteria in the uterus and necrotic tissue delays uterine involution and the repair of the endometrial. Furthermore, residual tissues may result in the cervix being held open and an increase in bacterial contamination of the endometrium. ${ }^{5,15}$ Indirectly, pregnancy length induced calving, twins, and stillbirth increase the occurrence metritis and endometritis by favoring the retention of fetal membranes. ${ }^{5}$

Metabolic stress: Failure of adequate calcium mobilization around the time of calving results in hypocalcaemia since calcium is an important element in the process of uterine involution reduces uterine contractions, causes dystocia, prolongs gestation any deficiency delays this process and is considered a risk factor for retained fetal membrane occurrence, it is a risk factor for metritis and endometritis occurrence. ${ }^{5,16}$

Negative energy balance: A negative energy balance (NEB) is often linked with severe and prolonged uterine inflammation and delayed uterine involution, which appears to be associated with a lack of bacterial killing after ingestion by phagocytes. NEB favors the development of many metabolic disorders, especially ketosis, which can increase the main important risk factors for metritis endometritis and retained placenta. ${ }^{17}$ At the start of lactation, dairy cows require a significant amount of glucose to produce lactose, and these requirements are maximal after the third week of postpartum. During this period, dairy cows have an insufficient appetite to consume the required energy since the diary animals have low food ingestion capacity. Energy deficiency results in lipid mobilization, metabolization in the liver as a source of energy and excessive production and accumulation of ketones, can reach toxic concentrations and reduce immune functions of the uterus by affecting phagocytosis and increase the risk of metritis and endometritis. $^{18}$

\section{Etiopathophysiology of Clinical Metritis and Endometritis}

The uterus is routinely contaminated with bacteria in the early postpartum period. ${ }^{19}$ Among the pathogenic bacteria that can affect the uterus and causes metritis and endometritis include Escherichia coli, Arcanobacterium pyogenes, Fusobacterium necrophorum, Bacteroides species, Staphylococcus species Pasteurella species, Haemophilussomnus, Pseudomonas aeruginosa, Clostridium species, and Streptococcus species. Among these, Arcanobacterium pyogenes, coliforms, and the Gram-negative anaerobes, Fusobacterium, and Bacteroides species are commonly encountered. ${ }^{20}$

The bacterial load in the uterus disrupts the normal uterine defense mechanism leading to life-threatening infection. The uterus after calving has damaged epithelial surface with fluids and debris from the tissues that facilitate the growth of the bacteria. ${ }^{19}$ Clinical metritis generally initiates with the establishment of the pathogenic organism to the uterine mucosa and their penetration through the epithelium and thereby releasing the toxins. ${ }^{6}$ Cows affected by endometritis have a very high concentration of bacterial lipopolysaccharide (LPS) and immune-depressant products that decrease the recruitment of leukocytes into the uterus to clear bacteria. The immunity female animals become weak and the species and load of the bacteria involved in the infection is a critical determinant for the establishment of endometrial infection. ${ }^{6,19}$

Elevated inflammation of metritis and endometritis results in impaired reproductive performance. ${ }^{21}$ These infections are believed to result in delayed uterine involution and damages to embryos, causing impaired or delayed fertility. Additionally, uterine infection and inflammation are associated with some alteration in the pattern of follicle growth on ovaries and disruption of subsequent luteal phase durations. ${ }^{6,21}$

Uterine diseases pathology extension of infection or inflammation to the oviduct likely disrupts the delicate balance of the immune systems that are required for fertilization. ${ }^{22}$ Bacterial infections disrupt the endocrine signaling in the hypothalamic-pituitary-gonadal axis and the secretion of gonadotrophins. Uterine infections disrupt ovarian follicle growth and function, with smaller and less steroidogenic ovarian follicles. ${ }^{21,22}$ Uterine infections reduce oocyte quality, with increased rates of meiotic arrest and germinal vesicle breakdown failure and cause infertility of female animals. $^{22}$

\section{Clinical Signs and Symptoms}

The most prominent abnormality observed in clinical metritis and endometritis are the yellowish mucopurulent discharged from the vulva opening. Pyrexia, systemic illness, dull, depressed with pale mucous membrane. Upon rectal examination the uterus having a doughy consistency and around the ovaries, the corpus luteum 
persistently can be palpated. ${ }^{8,9}$ Decreased milk yield, in appetence and increases infertility in dairy cows animals indicates uterine diseases. $^{23}$

\section{Diagnosis}

Among commonly used diagnostic tools, rectal palpation is probably the most common method for diagnosing uterine infections. ${ }^{24}$ The diagnosis of metritis and clinical endometritis should include inspection of the contents of the female genital tract by speculum or insertion of a clean-gloved-hand into the vagina. Manual examination of the vagina also facilitates the collection of fluid from the vagina to evaluate the presence and odour of pus, which can be used to score the severity of disease and predict the likely success of treatment. . $^{10,24}$

Vaginoscopy is a straightforward procedure that permits the evaluation of the characteristics of fluids in the anterior vagina and external cervical. Vaginal discharge can be scored and used as an indicator of uterine infection. ${ }^{3}$ Diagnosis of clinical endometritis in dairy cows; the presence of purulent uterine discharge or cervical diameter greater than $7.5 \mathrm{~cm}$ after 20 -days of postpartum or a mucopurulent discharge after 26-days postpartum defined clinically relevant endometritis. ${ }^{3,24}$

Ultrasound uterine measurement is convenient and allows for reliable result comparison. Ultrasonographic intrauterine fluid determination 3-weeks postpartum exhibits good sensitivity and specificity and is reliable for diagnosing endometritis. ${ }^{25}$

In the absence of pus cytological evidence of inflammation of the endometrium is associated with reduced fertility. ${ }^{21,25}$ Endometritis is characterized by inflammation of the uterine wall that results in a significant reduction in reproductive performance. The subclinical disease is defined by the proportion of polymorph nuclear neutrophils (PMNs) exceeding, usually about $5 \%$ of cells in samples collected by flushing the uterine lumen or by endometrial cytobrush in the absence of clinical metritis and endometritis. $^{24,25}$

\section{Therapeutics}

Non-steroidal anti-inflammatory drugs: Clinical metritis and endometritis can be treated by flunixin meglumine $1.1 \mathrm{mg} / \mathrm{kg}$ were administered intramuscularly twice a day for 3-days as anti-inflammatory, antipyretic and analgesic.

Intrauterine infusion: Treatment of metritis and other uterine diseases, different intrauterine therapies like antiseptics, antibiotics and immune modulators are infused into the uterus to eliminate the bacterial infection, stimulate the normal uterine defense mechanism, or to increase the blood flow to the uterus. The most routinely used intrauterine therapy is the infusion of iodine solution in water or saline. ${ }^{2}$

Systemic antibiotics: Broad-spectrum long-acting oxytetracycline at a dose of $20 \mathrm{mg} / \mathrm{kg}$ antibiotic can be given once intramuscularly to treat uterine bacterial disease infections. ${ }^{6}$ Penicillin is one of the most preferred antibiotics for postpartum metritis and endometritis because it penetrates all the layers of the uterus, and most of the bacteria penetrating the endometrium leading septicemia are responsive to penicillin. ${ }^{25,26}$ Additional antibiotics commonly used for the treatment of metritis and include third-generation cephalosporins, or a combination of ampicillin with oxytetracycline or cloxacillin. ${ }^{26,27}$

Hormones: It acts on the corpus luteum to cause luteolysis, forming a corpus albicans, and stopping the production of progesterone. ${ }^{4}$ Prostaglandin F2 alpha (PGF2 $\alpha$ ) and is particularly valuable when corpus luteum is not present and it is important to treat clinical metritis and endometritis if there is corpusluteum is detected during diagnosis. ${ }^{8,27}$

\section{Impact of Uterine Diseases in Production}

The clinical uterine disease has a marked impact on reproductive health in cattle, causing subfertility and infertility. Post-partum metritis caused subfertility by increasing the time to the first insemination by 7.2-days, reducing conception rate to the first insemination by $20 \%$, and increasing the calving to conception interval by 18.6-days. ${ }^{27,28}$ Clinical endometritis increased the interval to the first insemination by 11-days, and delayed conception by 32-days, compared with animals that did not have endometritis. Cows with clinical endometritis between 20 and 33-days postpartum were 1.7 times more likely to be culled for reproductive failure than cows without endometritis. ${ }^{24,28}$

Clinical metritis and endometritis negatively affect the performance of the world's dairy industry; economic losses are related to delay in the resumption of ovarian activity, increased number of services per conception, decreased milk yield, increased costs of treatment of the disease and cause the death of the productive animals from development of septicemia. ${ }^{29}$

\section{CONCLUSION AND RECOMMENDATIONS}

Clinical metritis and endometritis are the most frequent uterine disorders in dairy cows causing decreased fertility leading to high economic losses. These reproductive diseases of the uterus caused by multiple factors including poor nutrition, the season of the calving time, metabolic disturbance, dystocia, retained fetal membrane and non-specific diseases. This favors the entrance of pathogenic bacteria into the uterus and multiplication and development of weak immunity in the uterus. The uterus after calving delayed uterine involution and causes metritis and endometritis. Based on the above conclusions the following recommendation should be forwarded for veterinarian and production linked farmers. Accurate and early diagnosis and treatment of clinical metritis and endometritis is the key for future fertility in dairy herds. Supplement balanced feed containing minerals, vitamins, carbohydrates, and proteins for the production of good body condition and immunity to resist the occurrence of uterine disease in dairy cattle. Good management practice may be applicable in dairy farms to control and prevent any risk factors that disturb the normal physiology of female animals. 


\section{AKNOWLEGMENT}

My deepest heart thank goes to Department of Animal health Awi Zone, Livestock Resource Development and Promotion Office, Amhara region Ethiopia.

\section{REFERENCES}

1. Gilbert RO. Bovine endometritis: the burden of proof. Cornell Vet. 1992; 82: 11-14.

2. Deori S, Arundhati P. Bovine postpartum metritis and its therapeutics: A review. Indian Journal of Science and Technology. 2015; 8(23): 45. doi: 10.17485/ijst/2015/v8i23/52386

3. Gautam G, Nakao M, Yusuf K, Koike K. Prevalence of endometritis during post partum Period and its impact on subsequent reproductive performance in two Japanese dairy herds. Anim Reprod Sci. 2009; 116: 175-187. doi: 10.1016/j.anireprosci.2009.02.001

4. Wikipedia. Metritis. Web site. https://en.wikipedia.org/wiki/ Metritis. Accessed September 11, 2018.

5. Mounir A, Rachid K, Christian H, Gary CW. Risk factors of clinical and Subclinical endometritis in cattle: A review. Turkish Journal of Veterinary and Animal Sciences. 2017; 41: 1-11. doi: 10.3906/vet1603-63

6. Arundhat OI, Rahawy MA, Hadad JJ. Bacterial isolates associated with dystocia and Retained placenta in Iraqi buffaloes. Reprod Domest Anim. 2015; 43: 286-292. doi: 10.1111/j.14390531.2007.00892.x

7. Iain, MS, Sian EO. Postpartum uterine infection and endometritis in dairy cattle. Paper presente at: The $33^{\text {rd }}$ Annual Scientific Meeting of the European Embryo Transfer Association (AETE); Bath, United Kingdom; 2017.

8. Abdullah F, Chung EL, Abba Y, Tijjani A, Sadiq MA, Mohammed K, et al. Management of clinical case of endometritis in a cow: A case report. J Vet Adv. 2015; 5(4): 887-890. doi: 10.5455/ jva.20150425121148

9. Kahn C, Line S. The Merck Veterinary Manual. $9^{\text {th }}$ ed. Merial, USA: $2005 ; 334$.

10. Abdel MA, Emad S, Gohary EL, Gabr AA, Ahmed SA, Ebrahim SA, et al. Impact of supplementing propylene glycol and calcium propionate to primiparou buffalo cows during the late gestation and early lactation period on reproductive performance and metabolic parameters. Alexandria Journal of Veterinary Sciences. 2016; 51(1): 114-121. doi: 10.5455/ajvs.240341

11. Turk R, Samardzija M, Bacic G. Oxidative stress and reproductive disorders in dairy cows. In: Marek ER, ed. Dairy Cows: Nutrition, Fertility, and Milk Production. New York, USA: Nova Science Publishers; 2011: 57-98.
12. MacKay D, Miller AL. Nutritional support for wound healing. Altern Med Rev. 2003; 8: 359-377.

13. Chaffaux S, Lakdissi H, Thibier M. Epidemiology of clinical metritis and endometritis. Rec Med Vet. 1991; 167: 349-358.

14. Bruun J, Ersboll AK, Alban L. Risk factors for metritis in danish dairy cows. Prev Vet Med. 2002; 54: 179-190. doi: 10.1016/ s0167-5877(02)00026-0

15. Kim IH, Kang HG. Risk factors for postpartum endometritis and the effect of Endometritis on reproductive performance in dairy cows in Korea. J Reprod Develop. 2003; 49: 485-491. doi: $10.1262 /$ jrd.49.485

16. Laven RA, Peters AR. Bovine retained placenta: Etiology, pathogenesis, and economic loss. Vet Rec. 1996; 139: 465-471. doi: 10.1136/vr.139.19.465

17. Markusfeld O. Periparturient traits in seven high dairy herds. Incidence rates, Association with parity, and interrelationships among traits. J Dairy Sci. 1987; 70: 158-166. doi: 10.3168/jds. S0022-0302(87)79990-1

18. Suthar VS, Canelas-Raposo J, Deniz A, Heuwieser W. Prevalence of subclinical ketosis and relationships with postpartum diseases in European dairy cows. J Dairy Sci. 2013; 96: 2925-2938. doi: 10.3168/jds.2012-6035

19. Konigsson K, Gustafsson H, Kindah H. Ketodihdro-PGF2 $\alpha$, progesterone and uterine involution in primiparous cows with induced retained placenta and post partial endometritis. Reprod Domest Anim. 2002; 37: 43-51. doi: 10.1046/j.1439-0531.2002.00325.x

20. Paisley L, Micklesen WD, Anderson PB. Mechanisms and therapy for retained Membranes and uterine infections of cows: A review. Theriogenology. 1986; 25: 353-381. doi: 10.1016/0093691X(86)90045-2

21. Sheldon IM. Physiology and endocrinology symposium: Uterine infection: Linking infection and innate immunity with infertility in the high-producing dairy cow. J Anim Sci. 2015; 93: 2021-2033. doi: $10.2527 /$ jas.2014-8496

22. Marey MA, Yousef MS, Kowsar R, Hambruch N, Shimizu T, Pfarrer C. et la. Local immune system in oviduct physiology and pathophysiology: attack or tolerance. Domest Anim Endocrinol. 2016; 56(1): 204-211. doi: 10.1016/j.domaniend.2016.02.005

23. Geert O. Metritis and endometritis in high yielding dairy cows. Rev Bras Reprod Anim. 2015; 39(1): 164-172.

24. LeBlanc SJ, Duffield TF, Leslie KE, Batean KG, Keefe GP, Walton JS, et al. Defining and diagnosing postpartum clinical endometritis and its impact on reproductive performance in dairy cows. J Dairy Sci. 2002; 85: 2223-2236. doi: 10.3168/jds.S00220302(02)74302-6 
25. MSD Manual Veterinary Manual. Utrine diseases in dairy cattle. Web site. https://www.msdvetmanual.com/reproductive-system/ metritis-in-large-animals/acute- puerperal-metritis-in-large-animals. Accessed September 12, 2019.

26. Galvao KN. Identifying and treating uterine disease in dairy cows. Paper Presented at: The $47^{\text {th }}$ Florida Dairy Proceeding Conference; Gainesville, FL, USA. 2011.

27. Smith BI, Risco CA.Therapeutic and management options for postpartum metritis in dairy cattle. Comp Contin Educ Pract Vet.
2002; 24: 92-100.

28. Youngquist RS, Shore O. Postpartum uterine infections. In: Youngquist RS, ed. Current Therapy in Large Animal Theriogenology. Toronto, Canada: WB Saunders Company. 1997456.

29. Kasimanickam R, Kasimanickam V, Kastelic JP, Mucin and cytokines mRNA in endometrium of dairy cows with postpartum uterine disease or repeat breeding. Theriogenology. 2014; 81: 952-958. doi: 10.1016/j.theriogenology.2014.01.018 